\title{
Communication among the VLAN Settings and failure analysis Based on the port
}

\author{
Xiaoyan Liu ${ }^{a}$, Yuxia Yao ${ }^{b}$ \\ School of Changchun University of Science and Technology, Changchun 130600, China. \\ Mail_xiaoyan@yaho.com ${ }^{a}, 1072343548 @ q q . c^{b}{ }^{b}$
}

Keywords: Computer network, VLAN, Switch configuration, Failure analysis

\begin{abstract}
With application of expanding the range of VLAN technology, VLAN network become an important practice in the computer network curriculum project. Paper based on Packet tracer 6.0 operating platform, designed and implemented using layer 3 switches to realize communication between VLANS. At the same time analyzed the failure causes of in the process of communication. Help to better master the application and maintenance of VLAN environment.
\end{abstract}

\section{Introduction}

Along with the computer network application of constantly extend and expand, used to isolate the network broadcast storm between the VLAN technology is also widely used. In the process of computer network curriculum teaching in colleges and universities, VLAN network is a very important part in the practice teaching link training project in the computer network course [1]. This article uses the Packet Tracer6.0 simulation software design of the LAN based on layer 3 switches, to study the communication between the virtual local area network is realized by using layer 3 switches. Under the network environment and the typical port configuration of common faults in the communication process between vlans do the simple analysis, gives a method to detect and eliminate. At the same time simply summarizes the configuration model of troubleshooting steps.

\section{Packet Tracer6.0 profile}

Routing and switching is two very strong practicality courses in computer network. The traditional practice in the process of building environment, most used to purchase the corresponding routers, switches, PC unit into the corresponding training topology, for students to practice [2]. But due to the limitation of teaching funds, which makes laboratory equipment update cycle is long, the equipment quantity is limited, can't meet the needs of students training, to improve the students' learning effect is larger.

Packet Tracer6.0 simulation software was developed by cisco router switches, used for auxiliary teaching tool, for the network design, configuration, beginners to remove the network failure provides a virtual training environment[3]. The software interface is friendly, students can directly on the interface established by the method of drag and drop the topology of the network, the software is also provided to allow the student to the equipment configure IOS subset. Students can look for the network real-time operation by the software, and understand the packets in the network the marching process in detail.

\section{VLAN configuration form and typical failure analysis}

\section{Set in a layer 3 switching to implement the communication between vlans}

(1)Typical networking and Settings

Using layer 3 switches S3560 VLAN2 and communication between VLAN3, topology structure is shown in figure 1.

Work in simulation software topological area increase two models for $2950 \mathrm{t}-24$ switches (SwitchA and SwitchB), a layer 3 switches S3560, four computer model for PC - PT, According to 
the topology shown in figure 2.

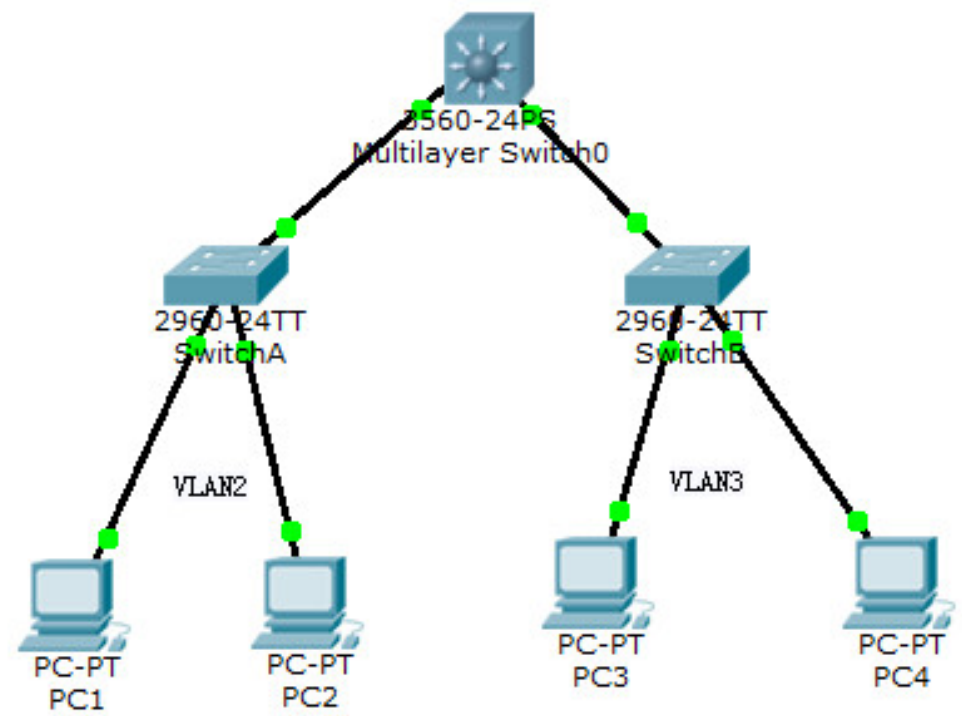

Figure 1 a three layer exchange to implement the communication between VLAN network diagram

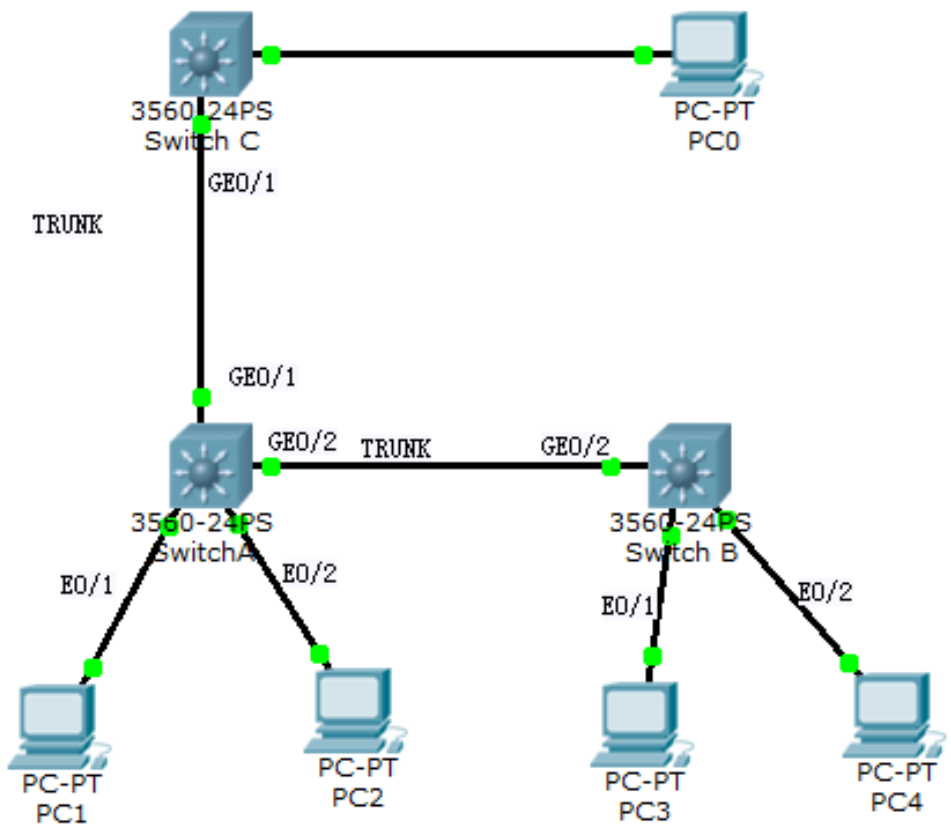

Figure 2 three layer 3 switching to implement the communication between VLAN network diagram

Respectively host configuration IP address and subnet mask. IP address and subnet mask as shown in table 1.

Host configuration method is: click the host PC1, in the open window, select the "desktop" TAB, click the "IP address configuration", select "manually" set up the corresponding IP address and subnet mask. Other hosts configuration in the same way.

Table 1 the host's IP address and subnet mask

\begin{tabular}{|l|l|l|}
\hline Computer name & IP address & subnet mask \\
\hline PC1 & 192.168 .1 .2 & 255.255 .255 .0 \\
\hline PC2 & 192.168 .1 .3 & 255.255 .255 .0 \\
\hline PC3 & 192.168 .10 .2 & 255.255 .255 .0 \\
\hline PC4 & 192.168 .10 .3 & 255.255 .255 .0 \\
\hline
\end{tabular}


By default, the host connected to the same switch form a VLAN1, the following is different in the same switch port on the division of VLAN.

Switch configuration method is: work in topology workspace, click on the switches in the dialog, click on the "command line" TAB, and enter the switch configuration interface, type the command under the command prompt.

SwitchA configuration is as follows:

SwitchA>enable

SwitchA\#configure terminal

SwitchA (config)\#vlan 2

SwitchA (config-vlan)\#exit

SwitchA (config)\#interface f0/2

SwitchA (config-if)\#switchport access vlan 2

SwitchA (config-if)\#interface f0/4

SwitchA (config-if)\#switchport access vlan 2

The same method configuration switches, B will PC3, and PC4 is added to the VLAN 3.

VLAN2 and VLAN3 use the layer 3 switche S3560 to communicate. Switch A and switch B connected to S3560 interface configured to TRUNK respectively. Configuration VLANIP S3560 and open three layer routing functions, which can realize VLAN2 and VLAN3.

Switches S3560 port F0/1 configuration is as follows:

Switch0(config-if)\#exit

Switch0(config)\#interface fastethernet0/1

Switch0(config-if)\#switchport mode trunk

Switch0(config-if)\#switchport trunk allowed vlan all

(2)The typical fault

Found in the configuration successful network environment between VLAN or VLAN internal users cannot access to each other.

(3)The failure analysis

VLAN inside can't communicate, first check the PC, and the light on the switch, if a VLAN interface in the UP state, then the light is on. The light, the interface is no problem. Secondly through the show VLAN command to see whether interface added to the corresponding VLAN. Find F0/1 port is still in the default VLAN1. Third, view of layer 3 switches and VLAN interface is set to the TRUNK, and layer 3 switches is open three layer routing functions.

(4)The troubleshooting

Divided port F0/1 on SwitchA mouth to VLAN2.

Set up three layer 3 switching to implement the communication between vlans

(1)Typical networking and Settings

Using the three layer 3 switches to realize communication between the vlans, topology structure is shown in figure 2. Work in simulation software topological area increase three layer 3 switches S3560 respectively named SwitchA, SwitchB and SwitchC, and computer models for PC - PT, According to the topology shown in figure 2.

With the above method to set the host IP address and subnet mask.

Three switches connected through the TRUNK. Configuration VLAN 3,4,5,6,7,100 on SwitchC. On SwitchC configuration VLAN 3,4,5,6,7,100, at the same time, configure corresponding three layer interface, including set GE0 / 1 TRUNK mode. On SwitchA configuration VLAN 3,4,100, at the same time setting GE0/1 and GE0/2 are in TRUNK mode. SwitchB configuration VLAN 5,6,7,100, including set GE0 /2 TRUNK mode. VLAN100 is management VLAN. Configuration TRUNK interfaces to allow all VLAN, specific command to set with the above.

(2)The typical fault

Configuration of success are found in the network environment between VLAN or VLAN internal users cannot access to each other. PC under SwitchA can communicate with PC which under SwitchC each other, but PC under SwitchB belong to VLAN5, 6, 7 cannot communicate with 
PC which under SwitchC each other. Under the VLAN 100 PC can communicate with PC under SwitchC.

(3)The failure analysis

First check the VLAN Settings is correct, on the premise of VLAN configuration failure analysis. Because between VLAN 100 PC can communicate with each other, so no fault in the circuit. Can communication between VLAN 100 PC, so the TRUNK transfers data to normal. Check SwitchB GE0/1 port state found that allow VLAN for 5,6,7 and 100 . Check SwitchA GE0/1 port and GE0/2 port state found that allows VLAN 3,4,100, no VLAN5, 6, 7. So VLAN5, 6, 7 data can't switch A. Because the mouth of the switches TRUNK configuration allows all VLAN, is to allow itself switches VLAN configuration in used, rather than allowing all VLAN in the whole topology.

(4)The troubleshooting

Increase VLAN5, 6, 7 in SwitchA.

\section{VLAN configuration troubleshooting steps}

\section{Check inside the VLAN interface state}

If network interface connection error, data cannot be passed, also cannot achieve communication. We can judge by looking at the network interface indicator method interface state. Network interface of the Link lights, said wiring success, when the lights flicker said data transmission.

\section{Check whether the VLAN division success}

The PC in the same VLAN can communicate with each other. The PC between different vlans can only be achieved by layer 3 switches or routers communicate with each other [4]. When PC cannot communicate with each other, through the command show vlan or Display a vlan to view whether port is added to the specified VLAN.

\section{Check whether to enable routing interfaces}

Realization of data transmission is decided by the state of the router interface. So in the network transmission problems are necessary to check the router port. First check on VLANIF interface IP address is configured properly. The second check router interface in the UP state. If the port is not in UP state, need to enable the interface. It is important to ensure that the interface is Administratively Down state, and the configuration has an IP address.

\section{Check the TRUNK port configuration}

In the virtual local area network (LAN) port have ACCESS and TRUNK two types. ACCESS port can only belong to a LAN, which transmits data packets from the same VLAN. The ports are used for the host to connect to the router or users. TRUNK port belongs to different virtual local area network, which can transmit multiple VLAN packets. The port general connect router or switch [5].

TRUNK interface often appear a problem is the relay model mismatch. For example a port mode of relay is closed, another relay mode to open ports. We can use the show interfaces trunk command to look at it. Another problem is allowed through virtual local area network on the list of problems, it is also the fault cases above problems.

Check whether the network segment is correct

The IP address in the same network segment belong to the same logical subnet, host can direct communication in the same logical subnet. The IP address of the PC should be directly connected to the VLAN router's address in the same network segment, the PC gateway address point to the router interface.

\section{Conclusion}

This article introduces the common of the two methods of VLAN configuration, and analyzes the common fault types, VLAN configuration and configuration fault detection method and solving measures. The article on troubleshooting steps is summarized. Due to the local area network architecture and many factors affect the normal operation of the complex and changeable, so in 
order to better realize the network communication, we need to constantly sum up the experiences of troubleshooting in the process of routine maintenance.

\section{References}

[1] Wei Wuhua. Higher vocational colleges and universities to explore the computer network curriculum teaching system [J]. Journal of shaanxi education [higher education], 2012 (9).

[2] Liu Ming. Based on the Packet tracer technology design and development of communication between the vlans [J]. Computer and digital engineering, 2014-7.

[3] Xu qiao, Zhao Jie. Based on the Packet Tracer experiment examination platform of network application and research [J]. Journal of Inner Mongolia normal university (education sciences), 2013, (3) : 66-72.

[4] Jiang Weixing. Intranet network security strategy based on VLAN [J] network security technology and application, 2009 (11) : 17-19

[5] Yang Jia. The application of VLAN technology in the local area network (LAN) [J]. Computer knowledge and technology (academic), 2007 (20). 